\title{
CARACTERIZAÇÃO E COMPARAÇÃO DA ABUNDÂNCIA E DIVERSIDADE DE PEIXES RECIFAIS EM DIFERENTES ENSEADAS DA ILHA DO ARVOREDO: CONSIDERAÇÕES SOBRE A “RESERVA BIOLÓGICA MARINHA (REBIOMAR) DO ARVOREDO"
}

\author{
IGOR EMILIANO G. PINHEIRO \& JORGE PABLO CASTELLO \\ Universidade Federal do Rio Grande - Laboratório de Recursos Pesqueiros Pelágicos, Rio Grande - RS - Brasil. CEP: 96201-900 - Caixa \\ postal: 474. oc.igoremiliano@gmail.com; docjpc@furg.br
}

\begin{abstract}
O trabalho visa caracterizar e comparar a abundância e diversidade das espécies de peixes recifais, visualmente acessíveis, em diferentes enseadas da llha do Arvoredo (27⒈'S; 48 $\left.22^{\prime} \mathrm{W}\right)$. A ilha não pertence integralmente a ReBioMar Arvoredo, portanto, as estimativas também foram comparadas entre áreas protegidas e não protegidas pela reserva. Os dados de abundância relativa, obtidos através de censos visuais, foram analisados através de técnicas univariadas e multivariadas. Os resultados mostram que não há grandes diferenças entre os diferentes pontos amostrais. Espécies como Anisotremus virginicus, Chaetodon striatus, Epinephelus marginatus, Haemulon aurolineatum, Micteroperca acutirostris, Pseudopeneus maculatus, Stegastes e Sparisoma sp. estão entre as mais abundantes e também entre as mais freqüentes na maioria das estações. A abundância geral e a diversidade foram mais influenciadas pela presença do banco de algas calcárias, existente em uma das estações, do que pela proteção assegurada pela reserva. Considerando-se a equitatividade e a comparação de espécies alvo da pesca comercial e esportiva (e.g. E. marginatus) observamos uma possível influência da ReBioMar Arvoredo.
\end{abstract}

PALAVRAS CHAVE: Reserva Biológica Marinha do Arvoredo, peixes recifais, Santa Catarina and censo visual.

\section{ABSTRACT}

Characterization and comparison of the abundance and diversity of reef fish in different bays of the Arvoredo Island: Considerations on the "Marine Biological Reserve (MarBioRe) of the Arvoredo"

A characterization and comparison of the abundance and diversity of reef fish species, visually accessible, in different bays of the Arvoredo Island $\left(27^{\circ} 17^{\prime} \mathrm{S} ; 48^{\circ} 22^{\prime} \mathrm{W}\right)$ was carried out. As the island does not belong entirely to the MarBioRe Arvoredo, the estimates were compared between protected and not protected areas. Relative abundance data, obtained through visual census, were analyzed using univariate and multivariate techniques. Results showed small differences between different sample stations. Most abundant and frequent species at the different sampling stations were Anisotremus virginicus, Chaetodon striatus, Epinephelus marginatus, Haemulon aurolineatum, Micteroperca acutirostris, Pseudopeneus maculatus, Stegastes and Sparisoma $s p$. The general abundance and diversity were more influenced by the presence of a coralline algae bank present at one of the sampling stations than by the protection ensured by the reserve. However, when dealing with the equitability and the comparison of fishery and sport target fishes (e.g. Epinephelus marginatus) we observed a possible influence of the MarBioRe Arvoredo.

KEYWORDS: Arvoredo Marine Biological Reserve, reef fish, Santa Catarina e visual census.

\section{INTRODUÇÃO}

Devido ao grande impacto antropogênico sobre os recursos renováveis e sistemas marinhos, onde a pesca é indicada como um dos principais modificadores (Roberts et al. 2005, Heithaus et al. 2008), as áreas marinhas protegidas (AMPs) tornaramse uma das principais ferramentas de conservação destes ambientes (Chape et al. 2005). Tais áreas são primordiais para a manutenção dos recursos pesqueiros e conservação da biodiversidade, além de proteger espécies residentes (Lubchenco et al. 2003) e seus estágios vulneráveis (Dayton 2000). Na ultima década, as diretrizes de manejo e conservação dos recursos marinhos tem enfocado as AMPs como ferramenta de manejo (Dayton et al. 2000, NRC 2001), baseado na quantidade substancial de evidências científicas que demonstraram respostas positivas de muitas espécies de peixes dentro das áreas protegidas da pesca (Côté et al. 2001, NRC 2001, Gell \& Roberts 2002, Halpern 2003).
No Brasil, dos estudos voltados para conservação e manejo de peixes recifais em AMPs, destacam-se os realizados por Ferreira \& Maida 2006, Floeter et al. 2006, Francini-Filho \& Moura 2008a,b. Em resumo, eles mostraram que nas áreas protegidas, quando comparadas aos pontos mais impactados, ocorrem indivíduos carnívoros predadores de topo em maior proporção e tamanho. No entanto, mesmo com as importantes contribuições destes estudos, o conhecimento a respeito da conservação dos peixes recifais na costa brasileira ainda é considerado restrito (Guimarães et al. 2001, Floeter et al. 2006).

$\mathrm{Na}$ região sul, onde há ilhas e arquipélagos costeiros pertencentes ao estado de Santa Catarina (SC), a carência de estudos a respeito de peixes recifais e da conservação e manejo em AMPs é mais evidente. Nesta região as ilhas possuem diversos status de conservação e utilização (e.g. Reserva Biológica Marinha do Arvoredo - que inclui as Ilhas do Arvoredo, Deserta, Galés e Calhau de São Pedro; 
Área de Proteção da Baleia; etc.), contudo, a fiscalização e gestão destas áreas ainda estão longe do ideal. Dos estudos realizados nesta região, podemos citar os levantamentos faunísticos (e.g. Lerner 1996, Wiggers 2003, Bouzon \& Freire 2007, Hostim-Silva et al. 2006, Koettker \& Freire 2006) e alguns trabalhos enfocando aspectos ecológicos (Blankensteyn et al. 2002, Bertoncini et al. 2003), biogeográficos (Floeter \& Soares-Gomes 1999, Floeter et al. 2001, 2004, 2005) e metodológicos (Godoy, et al. 2006). No entanto, publicações quantitativas da fauna e flora da região permanecem escassas e poucos são os estudos realizados na parte imersa destas ilhas.

Visando agregar conhecimento, este trabalho tem como objetivo estimar e comparar a abundância e diversidade das espécies de peixes recifais, visualmente acessíveis, em diferentes enseadas da
Ilha do Arvoredo e que são objeto de interesse para a pesca comercial e submarinha. Visto que esta ilha não pertence integralmente a ReBioMar Arvoredo, as estimativas também foram comparadas entre áreas protegidas e não protegidas pela reserva.

\section{MATERIAIS E MÉTODOS}

\section{Área de estudo}

A ReBioMar do Arvoredo (Figura 1) está localizada na zona costeira de Santa Catarina, a aproximadamente $11 \mathrm{~km}$ de Florianópolis. Apresenta uma área de 17.800 hectares e engloba as ilhas do Arvoredo, das Galés, a llha Deserta e Calhau de São Pedro (IBAMA 2004). Para realização deste estudo na referida unidade de conservação obtivemos junto ao IBAMA a licença ambiental número 02026000242/04-92.

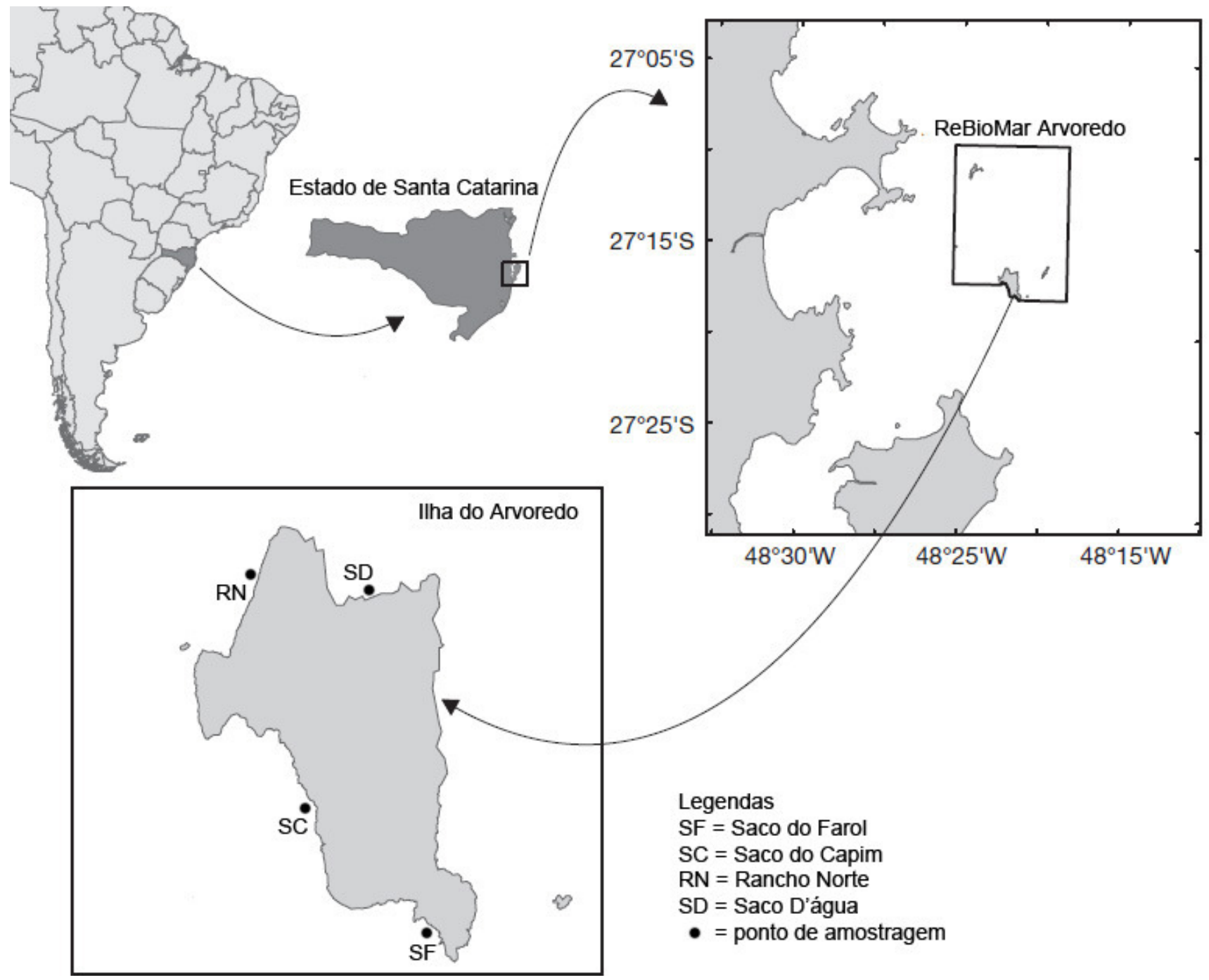

Figura 1: Localização da área de estudo e estações amostrais. 
Dentre as quatro ilhas a que mais se destaca é a do Arvoredo situada em $27^{\circ} 17^{\prime} 42^{\prime \prime}$ de latitude sul e 48 $22^{\prime} 36^{\prime \prime}$ de longitude oeste. O ambiente submarino da ilha do Arvoredo é essencialmente rochoso, sendo a maior parte constituída por rochas graníticas e areia, onde as profundidades variam de 0 a 30 metros (IBAMA 2004).

Oceanograficamente a região recebe influência de duas correntes oceânicas distintas: a relativamente quente e salgada corrente do Brasil Água Tropical (AT) - com baixa quantidade de nutrientes e a relativamente fria oriunda da corrente das Malvinas e misturada com aportes de águas continentais. Esta recebe o nome de Água Central do Atlântico Sul (ACAS) e é rica em nutrientes. As águas do estuário do Rio da Prata e da Lagoa dos Patos, também apresentam um importante papel na composição das águas costeiras superficiais desta região (Pimenta et al. 2004, Piola et al. 2005). De grande relevância para a produtividade da área em questão, é a presença da ACAS, durante os meses de primavera e verão. Nesta época, os ventos dominantes do NE favorecem a ocorrência da ressurgência costeira, possibilitando a elevação da ACAS do talude superior, para enriquecer a produtividade das regiões mais costeiras (Borzone et al. 1999).

\section{EXPEDIÇÕES}

Foram realizadas duas expedições a llha do Arvoredo; ambas no verão, por apresentarem condições mais favoráveis ao mergulho. Uma expedição piloto (04/02-06/03/2004) com 40 mergulhos, em diferentes profundidades e locais permitiu definir as estações amostrais, testar o método de amostragem e captar imagens para identificação das espécies. As imagens captadas auxiliaram na identificação de vinte e nove (29) diferentes famílias de Osteichthyes e uma (1) família de Chondrichthyes de acordo com Humann \& Deloach (2003) e Froese \& Pauly (2005).

Espécies das famílias Bleniidae, Gobiidae e Labrissomidae, que são de pequeno tamanho e de hábitos geralmente críptico, dificilmente se tornam alvos da pesca esportiva e/ou comercial e, portanto, não fizeram parte dos censos. Dessa forma, trinta famílias e quarenta e cinco espécies de peixes foram selecionadas para amostragem (Tabela 1). O gênero Stegastes (Família Pomacentridae) ocorre com 3 espécies na llha do Arvoredo (Hostim-Silva et al. 2006) com nichos, comportamento, aparência e morfologia semelhantes. Por esses motivos elas foram identificadas apenas ao nível de gênero. Da mesma forma, exemplares do gênero Sparisoma (Família Scaridae), que tem características taxonômicas que não são claramente visíveis (Feitosa et al. 2002) e que na llha do Arvoredo abrange 4 espécies (Hostim-Silva et al. 2006), foram também registradas ao nível de gênero. 
Tabela I: Famílias, espécies, nome comum, hábito de vida (H.V) das espécies determinadas para amostragem. Os H.V são: Epibentônico Ativo (E.A), discreto ou camuflado (D), bentônico, entocado (E) e territorialista (T). Espécies alvo da pesca (E.A.P), abundância relativa (A.R.) das espécies que ocorreram no Saco do Farol (SF), Saco do Capim (SC), Rancho Norte (RN) e Saco D'água (SD) e freqüência de ocorrência (FO).

\begin{tabular}{|c|c|c|c|c|c|c|c|c|c|c|}
\hline FAMÍLIAS & ESPÉCIES & Nome comum & H.V & E.A.P & SF & SC & $\mathrm{RN}$ & SD & A.R. & F.0\% \\
\hline Acanthuridae & Acanthurus chirurgus (Bloch, 1787) & Cirurgião & EA & - & - & - & 1,22 & - & 0,38 & 5 \\
\hline Apogonidae & Apogon pseudomaculatus (Longley, 1932) & Totó & $E$ & - & - & 0,36 & - & - & 0,08 & 5 \\
\hline Bothidae & Bothus ocellatus (Agassiz, 1831) & Solha / Linguado & D & - & - & 0,36 & - & - & 0,08 & 5 \\
\hline \multirow[t]{4}{*}{ Carangidae } & Seriola lalandi (Valenciennes, 1833) & Olho-de-boi & $P$ & $x$ & - & - & 12,71 & - & 3,95 & 15 \\
\hline & Seriola dumerili (Risso, 1810) & Olho-de-boi & $\mathrm{P}$ & $\mathrm{x}$ & - & 0,36 & 7,33 & - & 2,36 & 15 \\
\hline & Caranx crysos (Mitchill, 1815) & Xerelete & EA & $\mathrm{x}$ & 1,38 & 0,36 & 7,58 & - & 2,81 & 20 \\
\hline & Pseudocaranx dentex ( Bloch \& Schneider, 1801) & Garapoá & EA & - & 7,99 & - & 0,49 & - & 2,36 & 30 \\
\hline Centropomidae & Centropomus undecimalis (Bloch, 1792) & Robalo flecha & EA & $x$ & - & 2,19 & - & - & 0,46 & 10 \\
\hline Chaetodontidae & Chaetodon striatus (Linnaeus, 1758) & Borboleta listrado & EA & - & 7,16 & 4,38 & 12,96 & 4,43 & 7,83 & 85 \\
\hline Dactylopteridae & Dactylopterus volitans (Linnaeus, 1758) & Coió / Cabra-voadora & B & - & - & 0,73 & - & - & 0,15 & 10 \\
\hline Ephippidae & Chaetodipterus faber (Broussonet, 1782) & Enxada & EA & $x$ & - & - & - & - & 0,00 & 0 \\
\hline Fistularidae & Fistularia tabacaria (Linnaeus, 1758) & Peixe-corneta & EA & - & - & - & - & - & 0,00 & 0 \\
\hline \multirow[t]{3}{*}{ Haemulidae } & Haemulon aurolineatum (Cuvier, 1830) & Cotinga / Sapuruna & EA & $\mathrm{x}$ & 21,49 & 16,06 & 14,43 & 6,64 & 15,12 & 95 \\
\hline & Anisotremus virginicus (Linnaeus, 1758) & Salema / Mercador & EA & $x$ & 6,06 & 5,84 & 3,18 & 7,38 & 5,40 & 95 \\
\hline & Anisotremus surinamensis (Bloch, 1790) & Sargo-de beiço / Salgo & $\mathrm{E}$ & $\mathrm{x}$ & - & - & 1,47 & - & 0,46 & 20 \\
\hline Kyphosidae & Kyphosu ssp. & Pijirica / Piranjica & EA & $x$ & - & 1,09 & 0,49 & 3,69 & 1,14 & 35 \\
\hline \multirow[t]{3}{*}{ Labridae } & Bodianus pulchellus (Poey, 1860) & Budião-arara & EA & - & 0,28 & - & - & 1,11 & 0,30 & 15 \\
\hline & Bodianus rufus (Linnaeus, 1758) & Budião-papagaio & EA & - & - & - & - & 0,37 & 0,08 & 5 \\
\hline & Halichoeres poeyi (Steindachner, 1867) & Budião-rei & EA & - & 0,55 & 0,36 & - & - & 0,23 & 15 \\
\hline Lutjanidae & Lutjanus analis (Cuvier, 1828) & Cioba / Vermelho-caranha & EA & $x$ & - & 1,09 & 0,49 & 0,37 & 0,46 & 25 \\
\hline Malacanthidae & Malacanthus plumieri (Bloch, 1787) & Pirá & D & - & - & - & 0,24 & - & 0,08 & 5 \\
\hline Monacanthidae & Stephanolepis hispidus (Linnaeus, 1766) & Peixe-porco-galhudo & D & $x$ & - & 0,36 & - & - & 0,08 & 5 \\
\hline Mugillidae & Mugil curema (Valenciennes, 1836) & Parati & EA & $x$ & - & 0,36 & - & - & 0,08 & 5 \\
\hline Mullidae & Pseudupeneus maculatus (Bloch, 1793) & Salmonete / Saramunete & B & $\mathrm{x}$ & 7,71 & 9,12 & 7,09 & 16,24 & 9,57 & 100 \\
\hline Muraenidae & Gymnothorax funebris (Ranzani, 1839) & Caramuru / Moréia-verde & E & - & 0,28 & - & - & - & 0,08 & 5 \\
\hline Myliobatidae & Aetobatus narinari(Euphrasen, 1970) & Arraia-pintada / Raia-chita & EA & - & - & - & - & - & - & 0 \\
\hline Ogcocephalidae & Ogcocephalus vespertilio (Linnaeus, 1758) & Peixe-morcego & B & - & - & - & - & - & 0,00 & 0 \\
\hline Ophichthidae & Myrichthys ocellatus (Lesueur, 1825) & Falsa-moréia-pintada & B & - & - & - & - & - & 0,00 & 0 \\
\hline Ostracidae & Acanthostracion polygonius (Poey, 1876) & Peixe-cofre & D & - & 0,28 & - & 0,24 & - & 0,08 & 10 \\
\hline \multirow[t]{3}{*}{ Pomacanthidae } & Pomacanthus paru (Bloch, 1787) & Paru / Frade & EA & - & 0,55 & - & 1,96 & 1,48 & 1,06 & 40 \\
\hline & Holacanthus ciliaris (Linnaeus, 1758) & Peixe-anjo / Anjo-rainha & EA & - & - & - & - & - & 0,00 & 0 \\
\hline & Holacanthus tricolor (Bloch, 1795) & Tricolor / Vigário & EA & - & - & - & - & - & 0,00 & 0 \\
\hline \multirow[t]{3}{*}{ Pomacentridae } & Abudefduf saxatilis (Linnaeus, 1758) & Sargentinho & $E A$ & - & 2,75 & 1,82 & 0,98 & 6,64 & 2,81 & 60 \\
\hline & Chromis multilineata (Guichenot, 1853) & Mulata & $E A$ & - & 1,10 & 0,73 & - & 2,95 & 1,06 & 60 \\
\hline & Stegastes ssp. & Donzela & T & - & 3,03 & 6,20 & 3,42 & 17,71 & 6,84 & 100 \\
\hline \multirow[t]{2}{*}{ Scaridae } & Cryptotomus roseus (Cope, 1871) & Budião-rosa & B & - & - & - & - & - & 0,00 & 0 \\
\hline & Sparissoma ssp. & Papagaio & EA & - & 5,51 & 2,92 & 8,07 & 2,21 & 5,09 & 85 \\
\hline \multirow[t]{2}{*}{ Sciaenidae } & Odontoscion dentex (Cuvier, 1830) & Pescada-dentuda / Pirucaia & E & - & - & - & - & 0,37 & 0,08 & 5 \\
\hline & Pareques acuminatus (Bloch \& Schneider, 1801) & Maria-nagô / Listrado & E & - & 1,65 & 0,73 & - & - & 0,61 & 25 \\
\hline \multirow[t]{3}{*}{ Serranidae } & Serranus flaviventris (Cuvier, 1829) & Mariquita & E & - & - & - & - & - & 0,00 & 0 \\
\hline & Epinephelus marginatus(Lowe, 1834) & Garoupa-verdadeira & $\mathrm{E}$ & $\mathrm{x}$ & 2,48 & 3,28 & 10,27 & 13,65 & 7,37 & 95 \\
\hline & Mycteroperca acutirostris (Valenciennes, 1828) & Badelo-mira & EA & $x$ & 5,51 & 4,38 & 2,44 & 5,17 & 4,26 & 80 \\
\hline Sparidae & Diplodus argenteus (Valenciennes, 1830) & Marimbau & EA & $\mathrm{x}$ & 17,91 & 27,01 & 1,71 & 7,01 & 12,54 & 80 \\
\hline Synodontidae & Synodus synodus (Linnaeus, 1758) & Lagarto & B & - & 0,28 & 0,36 & - & - & 0,15 & 10 \\
\hline \multirow[t]{2}{*}{ Tetradontidae } & Canthigaster figueiredoi(Moura \& Castro, 2002) & Baiacu-mirim & EA & - & 0,55 & 0,36 & - & - & 0,23 & 15 \\
\hline & Sphoeroides spengleri (Bloch, 1782) & Baiacu-pimina & EA & - & 5,51 & 9,12 & 1,22 & 2,58 & 4,33 & 80 \\
\hline
\end{tabular}


Das quarenta e cinco espécies de peixes que fizeram parte deste estudo, dezesseis são alvos diretos da pesca esportiva, comercial ou da caça submarina. A determinação das espécies alvo da pesca foi feita segundo Carvalho-Filho (1999) e os hábitos de vida foram definidos segundo Humann \& Deloach (2003) e Smith (2004) (Tabela I). Os hábitos definidos são: bentônico (B), discreto ou camuflado (D), entocado (E), epibentônico ativo (E.A) e territorialista (T).

$\mathrm{Na}$ segunda expedição (13/01-16/02/2005) foram realizados 25 mergulhos para coleta dos dados. Apenas 20 (cinco para cada uma das quatro estações) foram usados nas análises, pois em cinco mergulhos as condições de visibilidade diferente poderiam afetar os resultados.

\section{ESTAÇÕES AMOSTRAIS}

Das 4 estações amostrais, no entorno da ilha do Arvoredo (Figura 1) determinadas com características contrastantes, duas encontram-se dentro da área protegida e duas fora dela.

Características como condições marinhas, visibilidade, tempo de mergulho e vento foram levadas em consideração visando que as réplicas fossem feitas em condições semelhantes. A profundidade de 10 metros foi demarcada como padrão entre as estações, com exceção da estação 3Rancho Norte (RN), onde os costões rochosos não ultrapassam o máximo de 9 metros de profundidade.

\section{Estação 1 - Saco do Farol (SF)}

O (SF) se encontra fora dos limites da reserva e apresenta substratos rochosos em profundidades de até 18 metros, sendo caracterizado por uma elevada heterogeneidade de nichos produzidos por rochas de tamanhos variados. Estas apresentam uma inclinação pequena, o que proporciona a presença de substrato consolidado por uma grande extensão (IBAMA 2004). Esta estação é abrigada de vento leste e nordeste, porém é totalmente desabrigada dos ventos do quadrante sul.

\section{Estação 2 - Saco do Capim (SC)}

Esta estação é protegida dos ventos nordeste e sudeste e se encontra fora dos limites da reserva. No (SC) o fundo é composto por rochas grandes repousadas de forma espaçada sobre fundo arenoso. As rochas são cobertas por diversos tipos de invertebrados sésseis e algas incrustantes, assim como em todas as estações, com poucas diferenças. O limite entre as rochas e areia é bem definido e não ultrapassa a profundidade de 14 metros. Nos fundos arenosos há poucas rochas esparsas que desaparecem com o aumento da profundidade. Esta região da ilha é bastante protegida de ondulações de leste e oferece uma boa alternativa para o desembarque em dias de ondulação sul.

\section{Estação 3 - Rancho Norte (RN)}

Estação dentro da área protegida apresenta costões rochosos relativamente rasos (máximo $9 \mathrm{~m}$ de profundidade), com rochas pequenas a médias e com uma heterogeneidade de nichos moderada. É importante ressaltar que os referidos costões fazem interface com o banco de nódulos de algas calcárias. Este banco caracteriza-se por um substrato ocupado por diferentes espécies de algas calcárias que proporcionam uma elevada heterogeneidade de microhabitats, o que por sua vez representa um grande potencial de abrigo para uma elevada diversidade de organismos (IBAMA 2004). Ressalta-se que os censos foram realizados no costão rochoso e não sobre o banco de algas. Esta estação é abrigada de todos os ventos com exceção do nordeste.

\section{Estação 4 - Saco D'água (SD)}

Também dentro da reserva, os costões rochosos desta estação têm seu limite com o substrato não consolidado localizado em uma profundidade que varia em torno de 15 metros (IBAMA 2004). O substrato é caracterizado por uma elevada heterogeneidade de nichos produzidos por rochas de tamanhos variados. Esta estação é protegida dos ventos do quadrante sul e oeste.

\section{AMOSTRAGEM}

A metodologia empregada foi primeiramente descrita por Brock (1954), e é aplicada para estimativas quali-quantitaivas, provendo informações a respeito da presença da espécie e abundância da população por unidade de área pesquisada.

Para todos os pontos fixados foi estabelecido um "trajeto fixo" a ser percorrido (De Girolamo \& Mazzoldi 2001, Godoy et al. 2006), indicado por uma trena com 25 metros de comprimento junto ao fundo.

As técnicas de censo visual geralmente subestimam o número de indivíduos de espécies bentônicas e daquelas de hábitos mais discretos com 
comportamento evasivo e de entocamento (Ackerman \& Bellwood 2000). O censo pode ser otimizado através da redução da velocidade de natação durante a pesquisa. No entanto, a baixas velocidades, os indivíduos das espécies epibentônicas mais ativas, podem ser contados mais de uma vez (Lincoln Smith 1988). Para minimizar esses efeitos, as contagens dessas espécies foram realizadas separadamente daquelas mais ativas e facilmente observáveis. Para as últimas foi utilizada uma maior velocidade de natação que para as espécies de hábitos mais discretos.

Para a amostragem, o mergulhador esticava a trena de 25 metros posicionando-se ao lado da linha, a uma distância perpendicular de 3 metros desta. Após um intervalo de 5 minutos, foram amostrados 75 metros quadrados para as espécies pelágicas e epibentônicas ativas. Ao fim do primeiro censo e de um novo intervalo de cinco (5) minutos, foram amostrados outros 75 metros quadrados para as espécies bentônicas, de hábitos discretos, territorialistas e entocadas. Todas as espécies de peixes escolhidas para amostragem foram censadas (Tabela 1). Os mergulhos tiveram uma duração efetiva média de trinta minutos e trinta segundos, divididos em dois minutos para esticar e recolher a trena, nove minutos para realização dos censos, sendo três minutos e trinta segundos para amostragem das espécies pelágicas e cinco minutos e trinta segundos para as bentônicas. O restante do tempo, dez minutos, foi de espera entre os censos.

As amostragens das estações foram realizadas em todos os dias em que houve condições favoráveis para o mergulho. Para cada estação foram considerados cinco mergulhos, totalizando vinte mergulhos para análise.

\section{ANÁLISE DOS DADOS}

Os dados de abundância, obtidos por intermédio do censo visual foram analisados através de técnicas univariadas e multivariadas.

Foi empregado 0 índice de diversidade Shannon-Wiener $\left(\mathrm{H}^{\prime}\right)$ (Shannon \& Weaver 1949), expresso por: $\mathrm{H}^{\prime}=-\sum p \cdot \ln (p)$. Onde $(p)$ é a proporção total da amostragem pertencente a cada espécie. A heterogeneidade foi calculada através do índice de Equitatividade (E) (Pielou 1974), de acordo com: $E=H^{\prime} / H_{\operatorname{máx}}$. Onde $H_{\text {max. }}=$ é a máxima diversidade de espécies, calculado pelo log2 (S). S é o total de espécies avistadas em cada estação.

Para comparação dos índices de diversidade de Shannon-Wiener $\left(\mathrm{H}^{\prime}\right)$ foi empregado o teste $t$ proposto por Hutcheson (1970) apud Magurran (1988). As comparações pareadas múltiplas foram feitas com a correção de Bonferroni (Zar 1984) para um nível de significância $\alpha=0,05$. Intervalos de confiança para os índices de diversidade e equitatividade obtidos foram calculados mediante o procedimento de bootstrap (Manly 1997). Para cada estação foram obtidas 1000 réplicas bootstrap, de $n$ igual à matriz original, que deram origem ao cálculo de 1000 novos índices de diversidade (H' e E).

Abundâncias relativas foram comparadas por meio de análises de variância de uma via (Zar 1984) com intervalo de confiança de 95\% $(\alpha<0,05)$. Havendo rejeição da hipótese nula $(\alpha=0,05)$ admitiuse a diferença e aplicou-se o teste de Tukey para discernir as diferenças entre os locais (CallegariJacques 2003). A normalidade foi testada através do teste de Kolmogorov-Lilliefors (Zar 1984) e a homocedasticidade foi verificada com 0 teste de Levene's (Hair et al. 1998). Naqueles casos em que os dados não se mostraram normais ou homocedásticos, estes foram transformados por log $(x+1)$ para assumirem os pré-requisitos para análise.

A fim de avaliar a similaridade entre as estações amostradas e verificar a determinação dos grupos hierárquicos foram geradas planilhas de similaridade, a partir dos dados quantitativos, usando o índice de Bray-Curtis. Posteriormente foram feitas análises de agrupamento (Hierarchical Cluster) utilizando o UPGMA ("Unweighted Pair-Group Mean Average"). Baseado nos grupos formados pelo Hierarchical Cluster foram geradas rotinas analíticas SIMPER (Similarity percentages - species contributions) para identificar as espécies que mais contribuíram para a dissimilaridade entre os grupos definidos para análise (Clarke \& Warwick 1994).

Foram consideradas como espécies mais representativas aquelas observadas em no mínimo quatro (4) réplicas em cada estação e como espécies mais freqüentes aquelas com freqüência de ocorrência (FO) superior a $80 \%$. 


\section{RESULTADOS}

\section{Composição geral e diversidade de espécies}

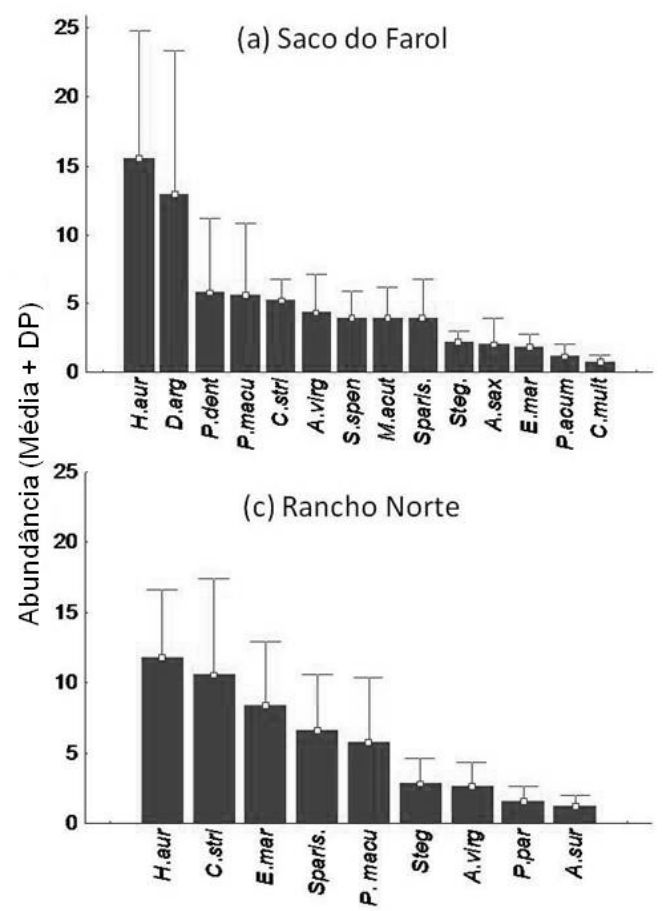

Os gráficos gerados com os valores das abundâncias das espécies mais representativas de cada estação são mostrados na figura 2.
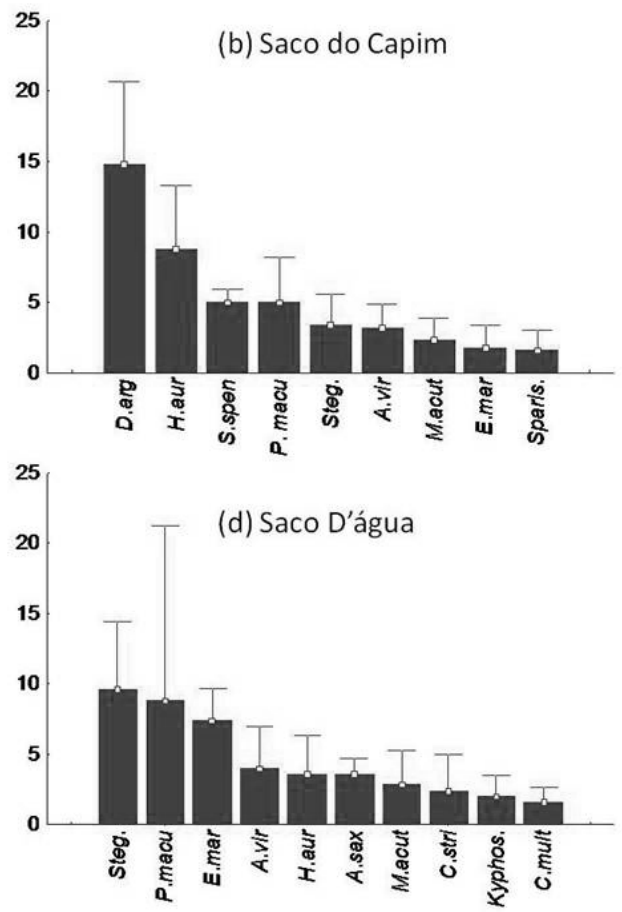

Figura 2: Abundância (média + desvio padrão) das espécies de peixes mais representativas nas 4 estações amostrais na llha do Arvoredo. A.sax = Abudefduf saxatilis; A.sur = Anisotremus surinamensis; A.vir $=$ Anisotremus virginicus; C.stri = Chaetodon striatus; C.mult = Chromis multilineata; D.arg = Diplodus argenteus; E.mar = Epinephelus marginatus; Kyphs. = Kyphosus; H.aur = Haemulon aurolineatum; M.acut = Mycteroperca acutirostris; P.acum = Pareques acuminatus; P.par = Pomacanthus paru; P.dent = Pseudocaranx dentex; P.macu = Pseudupeneus maculatus; Sparis. = Sparisoma; Steg. = Stegastes; S. spen = Sphoeroides spengleri.

Espécies como A. virginicus, E. marginatus, $H$. aurolineatum, $P$. maculatus e as do gênero Stegastes representaram, juntas em todas as estações, $44,3 \%$ da abundância total das espécies observadas. Espécies como C.striatus, M. acutirostris e o gênero Sparisoma que não foram representativas em todas as estações, mas em pelo menos 3 destas, abrangem 17\% da abundância total. $D$. argenteus, considerada como a espécie mais representativa nas estações fora dos limites da reserva, é a segunda espécie de maior abundância com 12,54\% da abundância relativa total.
Segundo a freqüência de ocorrência, as espécies mais freqüentes foram: $P$. maculatus (100\%), Stegastes (100\%), H.aurolineatum (95\%), A. virginicus $(95 \%)$, E. marginatus (95\%), C. striatus (85\%), Sparisoma (85\%), D.argenteus (80\%), $M$. acutirostris (80\%) e S.spengleri (80\%) (Tabela 1).

O maior índice de diversidade foi encontrado no Rancho Norte e as maiores equitatividades entre espécies no Saco D'água e Rancho Norte. Para à riqueza de espécies (S), o maior valor foi observado no Saco do Capim (26) seguido do Rancho Norte (22), Saco do Farol (21) e por último o Saco D'água (18) (Tabela 2).

Tabela II: Famílias, riqueza de espécies (S), índice de diversidade Shannon-Wiener $\left(\mathrm{H}^{`}\right)$ e Equitatividade (E).

\begin{tabular}{lllll}
\hline Estação & Famílias & S & E & H' $^{\prime}$ \\
\hline Saco do Farol & 15 & 21 & 0,8171 & 2,488 \\
\hline Saco do Capim & 19 & 26 & 0,7549 & 2,46 \\
\hline Rancho Norte & 15 & 22 & 0,842 & 2,603 \\
\hline Saco D'água & 12 & 18 & 0,8588 & 2,482 \\
\hline
\end{tabular}


Os intervalos de confiança para os índices de diversidade e equitatividade são apresentados na figura 3.

Para o índice de diversidade (Figura 3a) observa-se que os intervalos de confiança diferem pouco entre as diferentes estações, com exceção do RN que tem seu intervalo de confiança formado por valores mais altos. A diferença com relação ao $\mathrm{RN}$, apesar de pouco evidente, mostra a influência do banco de algas calcárias na diversidade de peixes. Já para a equitatividade (Figura $3 b$ ) nota-se que os intervalos de confiança com valores mais altos ocorrem nas estações dentro dos limites da reserva.
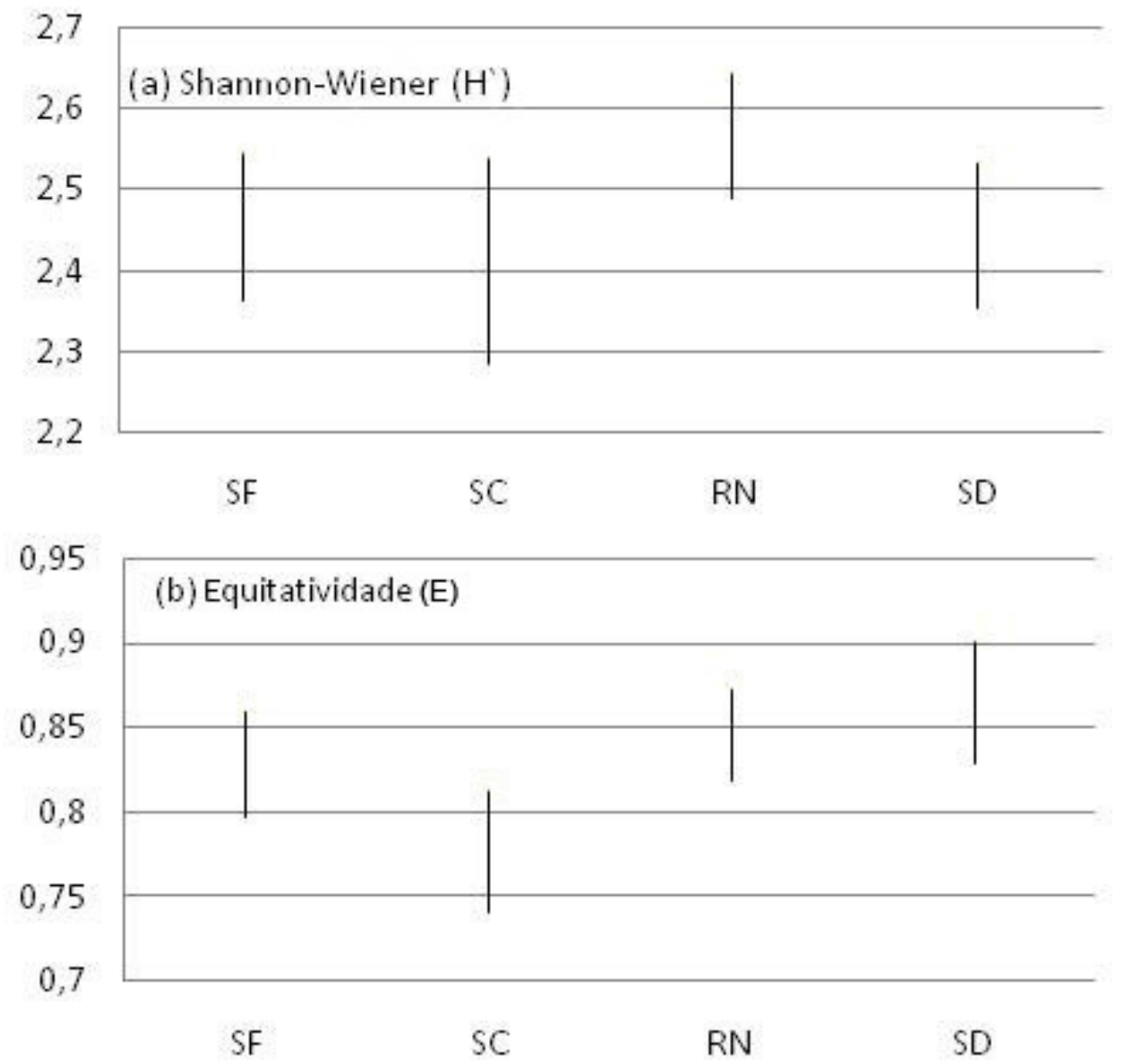

Figura 3: Intervalos de confiança construídos através de 1000 permutações Bootstrap para o índices de diversidade de Shanno-Wiever ( $\mathrm{H}^{\prime}$ ) e equitatividade (E) das estações amostrais ( $\mathrm{SF}=\mathrm{Saco}$ do Farol, SC= Saco do Capim, RN= Rancho Norte e SD= Saco D'água) na llha do Arvoredo.

As comparações do índice de Shannon-Wiener $\left(H^{\prime}\right)$ entre as estações feitas através do teste $t$ proposto por Hutcheson e corrigida pela correção de Bonferroni (onde $p$ deve ser menor que 0,0083 para se rejeitar a hipótese $\mathrm{H}_{0}$ ) não detectou diferenças significativas (Tabela 3 ), demonstrando semelhança entre as estações amostrais.

Tabela III: Resultados da comparação da diversidade entre as estações amostrais (SF= Saco do Farol, SC= Saco do Capim, RN= Rancho Norte e SD= Saco D'água) realizada através do teste t proposto por Hutcheson e corrigida pela correção de Bonferroni.

\begin{tabular}{lllll}
\hline & SF & SC & RN & SD \\
\hline SF & & 0,574 & 0,059 & 0,892 \\
\hline SC & 0,574 & & 0,036 & 0,652 \\
\hline RN & 0,059 & 0,036 & & 0,042 \\
\hline SD & 0,892 & 0,652 & 0,042 & \\
\hline
\end{tabular}




\section{COMPARAÇÕES DA ABUNDÂNCIA E ANÁLISE DE} SIMILARIDADE

Quando comparada a abundância total das espécies entre as estações, não foram constatadas diferenças significativas $(p=0,2522)$. $O$ mesmo aconteceu quando foram comparadas áreas protegidas e não protegidas ( $p=0,7205)$.

Das 16 espécies que são alvo da pesca (Tabela 1), P. maculatus, A. virginicus, M. acutirostris, $H$. aurolineatum, $E$. marginatus e $D$. argenteus foram observadas em todas as estações possibilitando comparações de suas abundâncias entre estações e entre áreas protegidas e não protegidas.
A comparação de abundância de $P$. maculatus nas diferentes estações e em áreas protegidas e não protegidas (Figura 4a) não mostrou diferenças significativas entre estações $(p=0,9966)$ e entre área protegida e não protegida $(p=0,8098)$. O mesmo aconteceu para o $A$. virginicus (Figura $4 b$ ) e $M$. acutirostris (Figura 4c) com $p=0,6718$ e $p=0,5498$ respectivamente para comparação entre estações e $p=0,6531$ e $p=0,4341$ respectivamente para a comparação entre áreas protegidas e não protegidas. (a) Pseudupeneus maculatus

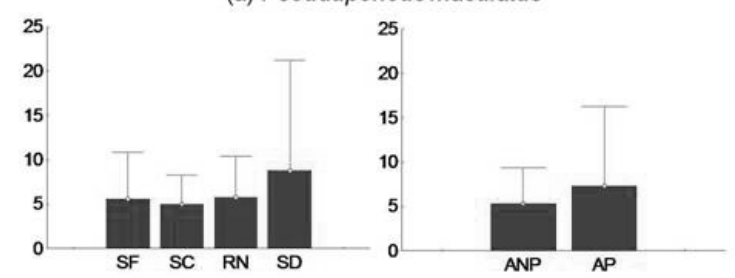

(c) Mycteroperca acutirostris

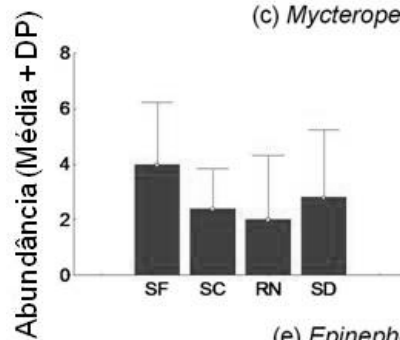

(e) Epinephelus marginatus

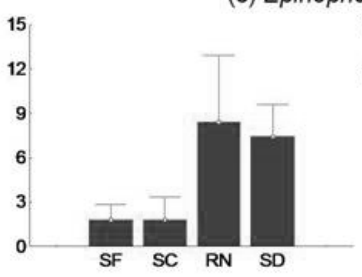

Estações
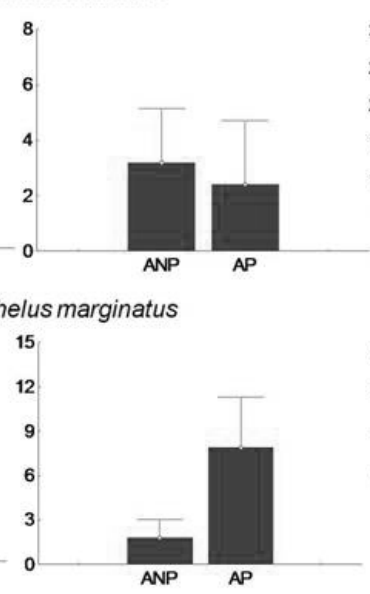

Fator Proteção

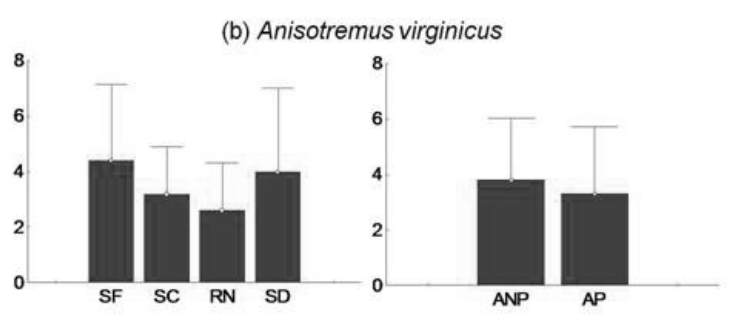

(d) Haemulon aurolineatum

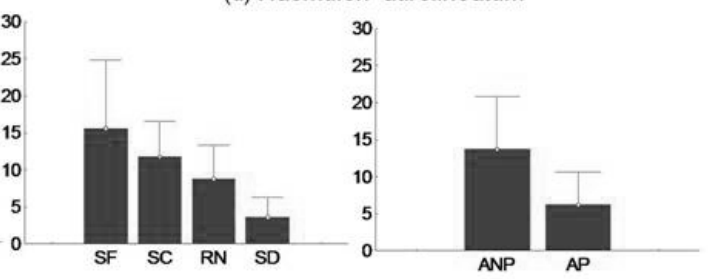

(f) Diplodus argenteus

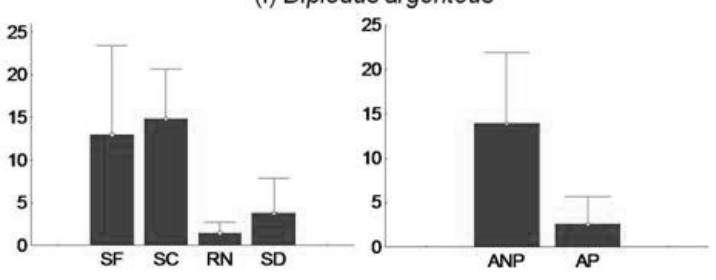

Estações

Fator Proteção

Figura 4: Abundância (média + desvio padrão) das espécies que são alvo da pesca considerando-se as 4 estações amostrais (SF= Saco do Farol, $S C=$ Saco do Capim, $R N=$ Rancho Norte e $S D=$ Saco D'água) e o fator proteção ( $\mathrm{ANP}=$ área não protegida e $\mathrm{AP}=$ área protegida).

Para $H$. aurolineatum (figura 4d) a comparação de abundância nas diferentes estações constatou diferenças significativas $(p=0,04319)$. O teste de Tukey identificou que a diferença ocorreu entre o Saco do Farol e o Saco D'água. Porém, quando a comparação da abundância foi realizada entre áreas protegidas e não protegidas não foi verificada diferença significativa $(p=0,1686)$.
Para E. marginatus (Figura 4e) e D. argenteus (Figura 4f) as comparações de abundâncias entre estações indicaram diferenças significativas em ambas as espécies ( $p=0,00068$ e $p=0,002$ respectivamente). No caso do E. marginatus, o teste de Tukey verificou diferença entre as estações dentro e fora dos limites da reserva. Analogamente, constatou-se o mesmo para $D$. argenteus, com exceção do Saco D'água que não apresenta 
diferença significativa se comparado com Saco do Farol. Na comparação da abundância entre áreas protegidas e não protegidas também foram observadas diferenças significativas para $0 E$. marginatus e $D$. argenteus $(p=0,00002$ e $p=0,0001$, respectivamente).

A análise de agrupamento (Figura 5), gerada com os dados de abundância das espécies, separou
3 grupos: o número 1, com maior similaridade, é formado pelas duas estações que se encontram fora dos limites da reserva, o grupo 2 e 3 formados por estações que se encontram dentro dos limites da reserva. O grupo 3 que inclui o Rancho Norte é o menos similar aos demais grupos.

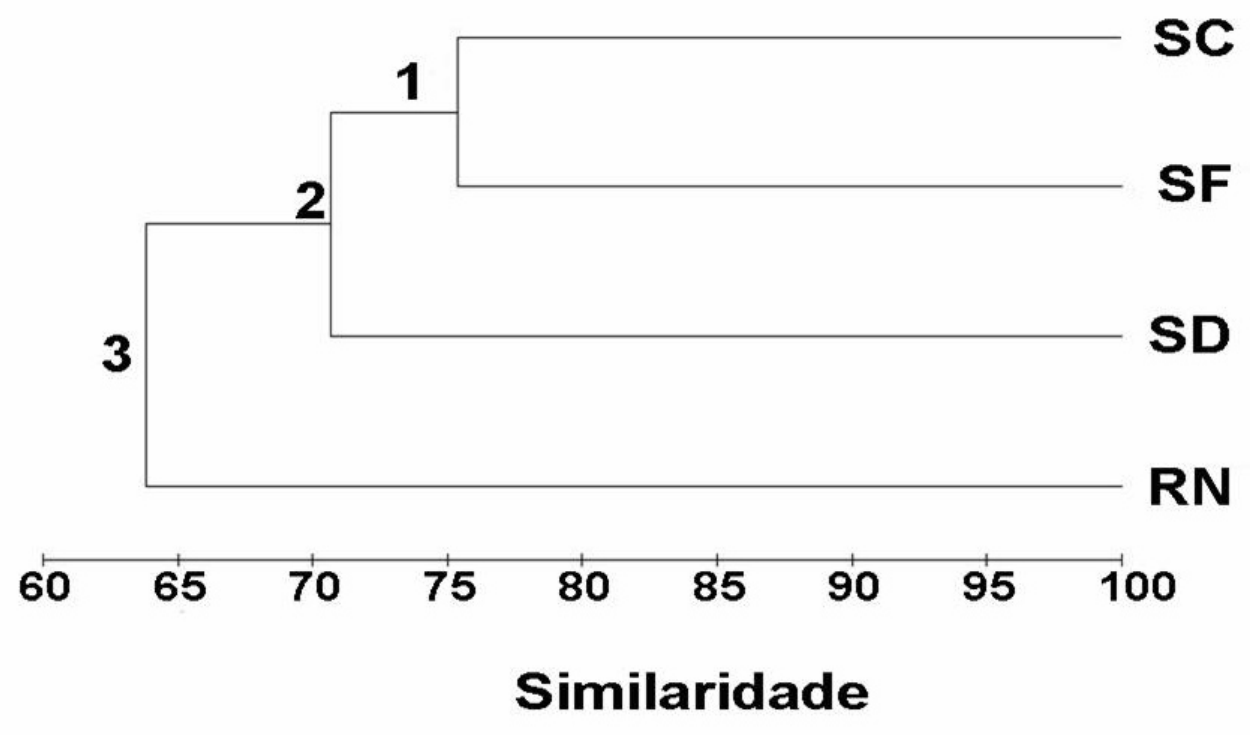

Figura 5: Analise de agrupamento gerada com os dados de abundância das 4 estações amostrais (SF= Saco do Farol, $\mathrm{SC}=$ Saco do Capim, $\mathrm{RN}=$ Rancho Norte e $\mathrm{SD}=\mathrm{Saco}$ D'água) na llha do Arvoredo.

A rotina analítica de SIMPER mostrou que as espécies que mais contribuíram para a dissimilaridade com relação à abundância entre o grupo 1 e 3 foram: $D$. argenteus (17,03\%), S. lalandi $(14,11 \%)$, C. striatus $(9,34 \%)$ e E. marginatus (8,95\%). Já com relação aos grupos 3 e 2, a análise mostrou que as espécies que mais contribuíram para a dissimilaridade foram: Seriola lalandi (14,61\%), Chaetodon striatus (11,52\%) e Haemulon aurolineatum $(11,52 \%)$.

Por ultimo, a rotina analítica de SIMPER aplicada aos grupos 1 e 2 mostrou que as espécies que mais contribuíram para a dissimilaridade na abundância foram: $D$. argenteus $(18,62 \%), H$. aurolineatum $(15,29)$ e E. marginatus $(10,26 \%)$.

\section{DISCUSSÃO}

Espécies como A. virginicus, C. striatus, $E$. marginatus, $H$. aurolineatum, $M$. acutirostris, $P$. maculatus, Stegastes e Sparisoma estão entre as mais abundantes e também entre as mais frequentes na maioria das estações.

Espécies de maior abundância, tanto em áreas protegidas e não protegidas, como $\mathrm{H}$. aurolineatum e D. argenteus, têm hábitos gregários (Humann \& Deloach 2003, Hostim-Silva et al. 2006) e, por este motivo, apresentam maior variabilidade em suas abundâncias. Ambas as espécies tiveram abundância mais elevada em regiões fora dos limites da reserva. No caso do $P$. maculatus, observa-se alta variabilidade na sua abundância apenas no SD o que pode ser explicado pela observação de 32 indivíduos jovens ao longo do transecto. Este fato aumentou o desvio padrão da espécie nesta estação. As demais espécies observadas apresentam variabilidades na abundância, no entanto menos evidentes. As características biológicas e comportamentais dos peixes são responsáveis por essa variabilidade 
observada em estudos ecológicos. A única maneira para reduzir esta variabilidade seria o aumento no número de réplicas (Godoy et al. 2006).

Nossos resultados indicam que entre as 4 estações amostrais existem poucas diferenças no que diz respeito à abundância geral e diversidade de espécies. Em parte essas diferenças pouco pronunciadas podem estar relacionadas às semelhanças existentes entre as 4 estações amostrais (profundidade e costões rochosos que fazem interface com a planície arenosa) e também pelo fato de que boa parte das espécies censadas não são alvos específicos da pesca. Segundo Micheli et al. (2004), os padrões espaciais da abundância de peixes que não são alvos da pesca não são diretamente afetadas pela reserva, embora efeitos indiretos possam ocorrer (e.g. efeito cascata). Tais fatores podem ter atenuado as diferenças entre as estações e entre áreas com diferentes regimes de proteção.

A estação que apresentou a maior disparidade, tanto para a abundância como para a diversidade foi o RN. Tal constatação pode estar relacionada à proximidade da estação amostral com o banco de algas calcárias existente nesta região. Bancos de algas calcárias suportam uma grande diversidade de organismos associados. Tais bancos proporcionam uma elevada heterogeneidade de micro-habitat, pois propiciam substratos duros para fixação de invertebrados marinhos e um grande potencial de abrigo para diversos organismos (Foster 2001, Gherardi 2004).

É importante ressaltar que o $C$. striatus espécie predadora de invertebrados bentônicos (Humann \& Deloach 2003, Hostim-Silva et al. 2006) teve a maior média de abundância na estação RN, provavelmente pela influencia do banco de algas calcárias. Adicionalmente, há também espécies de passagem como S. lalandi, vista unicamente sempre em grupos de mais de cinco indivíduos no RN, que podem usar esta área como área de alimentação. Ambas as espécies tiveram importância considerável para que o $\mathrm{RN}$ fosse à estação menos similar as demais. Outro fator que contribui para a maior dissimilaridade desta estação é sua posição em relação à ação das ondas e ventos. Segundo Friedlander et al. (2003) a biomassa dos peixes e o número de indivíduos é maior em regiões menos expostas a ação das ondas, e o RN é, dentre as estações amostradas, a que se encontra mais protegida desta ação.

Estações com regime de proteção têm influência sobre os índices de equitatividade, pois os intervalos de confiança diferem consideravelmente entre as estações protegidas e não protegidas pela ReBioMar. O regime de proteção pode estar favorecendo uma distribuição mais homogênea das abundâncias das espécies.

Apesar da semelhança na abundância geral e diversidade entre as estações, na comparação das espécies que são alvos da pesca algumas diferenças significativas puderam ser observadas. Assim, para H. aurolineatum, a comparação entre o SF (maior abundância) e o SD (menor), a diferença significativa pode ser atribuída aos regimes de proteção distintos. Esta espécie, tende a se manter afastada quando os mergulhadores se aproximam, sendo necessária uma aproximação cautelosa (Humann \& Deloach 2003, Hostim-Silva et al. 2006). Porém, o encontro com mergulhadores é algo freqüente $e$, portanto, é provável que esta espécie se mostre menos evasiva, aumentando sua abundância no SF e SC, onde as operações de mergulho são freqüentes, principalmente no verão. A rotina analítica SIMPER aplicada para verificar a dissimilaridade entre os grupos formados na Figura 5 enfatiza essa influência, visto que esta espécie contribui com $15,26 \%$ da dissimilaridade entre os grupos 1 (formado pelas estações SF e SC) e 2 (Estação SD). No entanto, na comparação das abundâncias em estações protegidas com aquelas sem proteção para $H$. aurolineatum essas diferenças não foram encontradas.

Para $D$. argenteus, segunda espécie de maior abundância relativa total, foram constatadas diferenças significativas entre as estações fora dos limites da reserva (SF e SC - grupo 1) e o RN (grupo 3). Esta espécie é avistada freqüentemente em águas turbulentas (Humann \& Deloach 2003, Hostim-Silva et al. 2006). A estação RN, a mais protegida da ação das ondas e com águas mais calmas acarretou uma menor abundância desta espécie. Soma-se a isso a presença do banco de algas calcárias, visto que esta espécie tem hábito de viver sobre fundos rochosos ou arenosos (Humann \& Deloach 2003, Hostim-Silva et al. 2006). A análise 
SIMPER também mostra a influência destes fatores sobre esta espécie (dissimilaridade igual $17 \%$ entre 0 grupo 1 e 3$)$.

É com E. marginatus (garoupa), espécie altamente comercial (Froese \& Pauly 2005), que a influência do fator proteção se tornou mais evidente. A rotina analítica SIMPER reafirma esta influência visto que esta espécie contribui com $8,95 \%$ da dissimilaridade entre os grupos 1 e 3 e $10,26 \%$ entre os grupos 1 e 2 . A garoupa merece atenção em especial, pois tem grande importância para a pesca artesanal no estado de Santa Catarina (Medeiros et al. 1997) e é para a pesca subaquática um dos alvos mais comuns (Carvalho-Filho 1999, Hostim-Silva et al. 2006). Na llha do Arvoredo, durante a realização dos censos, foram observados pequenos barcos, que vão a ilha com intuito de praticar pesca subaquática, sendo a garoupa um alvo muito apreciado. A pesca subaquática pode ser praticada, em regiões fora dos limites da reserva, a uma distância de $100 \mathrm{~m}$ da ilha. Este pode ser o motivo pela qual a garoupa se encontra em menor número nessas áreas. A espécie é hermafrodita protândrica e pode ser mais sensível a pressão de pesca (Falcón et al 1996), estes fatores podem acarretar no aumento da abundância da garoupa em áreas protegidas.

Para P.maculatus, A.virginicus e M.acutirostris não foram verificadas diferenças significativas entre estações nem entre áreas protegidas $\mathrm{e}$ desprotegidas. Esta constatação pode estar associada às características destas espécies e a diferente pressão de pesca exercida sobre elas.

A P. maculatus tem importante valor comercial (Froese \& Pauly 2005), no entanto, a pesca dirigida a esta espécie é artesanal (Cervigón et al. 1992) e ocorre em profundidades maiores do que a área amostrada no presente trabalho. Por este motivo, as médias de abundância desta espécie não diferem significativamente.

Já $A$. virginicus é facilmente encontrada nas Ilhas da ReBioMar, tanto em locais abrigados como aqueles expostos à ação das ondas (Hostim-Silva et al. 2006). Adicionalmente, ela não é alvo preferencial da pesca comercial e subaquática (Froese \& Pauly 2005). Estes fatores podem atenuar as diferenças na abundância entre as estações e entre áreas protegidas e desprotegidas.
Por fim, M. acutirostris, de alto interesse comercial e comparável à garoupa (Froese \& Pauly 2005), não teve sua abundância influenciada pelo regime de proteção. No entanto, a pressão de pesca exercida sobre o M. acutirostris é menor. Somando-se a isso, os exemplares desta espécie costumeiramente deixam-se levar pelas correntes próximas ao fundo (Humann \& Deloach 2003), além de ser esta, dentre os serranídeos, a espécie que apresenta hábitos de vida mais versáteis e ativos (Gibran 2007). Estes fatores podem proporcionar ampla distribuição a $M$. acutirostris na llha do Arvoredo dentro da isóbata amostrada.

Através deste primeiro estudo quantitativo dos peixes recifais da llha do Arvoredo, concluímos que não há grandes diferenças na abundância geral e na diversidade entre as diferentes enseadas desta Ilha. No entanto, se considerarmos a equitatividade e a comparação de espécies alvo da pesca notamos algumas diferenças que podem estar associadas à influência da proteção assegurada pela ReBioMar Arvoredo. Contudo, este estudo não teve como objetivo avaliar a efetividade desta reserva para fins de conservação, mas sim trazer uma primeira avaliação quantitativa dos peixes recifais da região em questão. Conclusões robustas sobre a importância e benefícios aportados por esta UC devem ser obtidas com uma maior cobertura temporal, maior número de observações e de pontos amostrais. Portanto, enfatizamos a necessidade da realização de novos estudos que compreendam tópicos como padrões temporais e espaciais da ictiofauna; assim como avaliações da complexidade estrutural e do gradiente de tamanho e abundância de peixes através dos limites da reserva, para que assim possa se elucidar de maneira mais precisa os benefícios assegurados pela ReBioMar do Arvoredo.

\section{AGRADECIMENTOS}

Agradecemos ao Project AWARE pelo apoio financeiro, a Marinha do Brasil e a Capitania dos Portos de Santa Catarina por ter autorizado o desembarque e permanência na Ilha do Arvoredo. Agradecemos também ao IBAMA por ter cedido à autorização para pesquisa na Reserva Biológica Marinha do Arvoredo e pelo suporte prestado. Pelas importantes recomendações e sugestões agradecemos ao Dr. Jose Angel Alvarez Perez. 


\section{REFERÊNCIAS}

ACKERMAN, JL \& DR BELLWOOD. 2000. Reef fish assemblages: A re-evaluating using enclosed rotenone stations. Mar. Ecol. Prog. Ser., 206: 227-237.

BERTOCINI, AB, LF MACHADO, M HOSTIM-SILVA \& JP BARREIROS. 2003. Reproductive Biology of the Dusk Grouper Epinephelus marginatus (Lowe, 1834). Braz. Arch. Biol. Tech., 46: 373-381.

BLANKENSTEYN, A, FS ALMEIDA, CF GUIMARAES, JB BOUZON, LJ WEISS, LM CAMARGO, S TELLES \& AS FREIRE. 2002. A macrofauna bentônica do banco de algas calcarias da Reserva Biológica Marinha do Arvoredo, SC, Brasil, pp. 83-93. In: Anais do $2^{\circ}$ Simpósio de Áreas Protegidas. Conservação no Âmbito do Cone Sul. Universidade Católica de Pelotas, RS.

BORZONE, CA, PR PEZZUTO \& E. MARONE. 1999. Oceanographic characteristics of a multispecific fishing ground of the Central South Brazil Bight. Mar. Ecol., 20 (2): 131-146.

BOUZON, JL \& AS FREIRE. 2007. The Brachyura and Anomura fauna (Decapoda: Crustacea) in the Arvoredo Marine Biological Reserve, southern Brazilian coast. Braz. J. Biol., 67: 631-637.

BROCK, VE. 1954. A preliminary report on a method of estimating reef fish populations. J. Wild. Manag., 18: 297-308.

CALLEGARI-JACQUES, SM. 2003. Bioestatística: princípios e aplicações. Porto Alegre, Artmed. 255p.

CARVALHO-FILHO, A. 1999. Peixes costa brasileira. 3ed. São Paulo, Marca D'Agua. 320p.

CERVIGÓN, F, R CIPRIANI, W FISCHER, L GARIBALDI, M HENDRICKX, AJ LEMUS, R MÁRQUES, JM POUTIERS, G ROBAINA, B RODRIQUEZ. 1992. Fichas FAO de identificación de especies para los fines de la pesca. Guia de Campo de las especies comerciales marinas y de águas salobres de la septentrional de Sur América. Roma, FAO, 1992. 513p.

CHAPE S, J HARRISON, M SPALDING \& I LYSENKO. 2005. Measuring the extent and effectiveness of protected areas as an indicator for meeting global biodiversity targets. Philos. Trans. R. Soc. Lond., 360: 443-455.

CLARKE, KR \& RW WARWICK. 1994. Change in marine communities: an aproach to statistical analysis and interpretation. Bournemouth, Bourne. $859 p$.

CÔTÉ, IM, I MOSQUERA, \& JD REYNOLDS. 2001. Effects of marine reserve characteristics on the protection of fish populations: a meta-analysis. J.Fish Biol., 59 (Supplement A):178-189.

DAYTON, PK, E SALA, MJ TEGNER \& SF THRUSH. 2000. Marine protected areas: parks, baselines, and fishery enhancement. Bull. Mar. Sci., 66: 617-634.

DE GIROLAMO M \& C MAZZOLDI. 2001. The application of visual census on Mediterranean rocky habitats. Mar. Env. Res., 51: 1-16.

FALCÓN, MJ, SA BORBONE, A BRITO \& CM BUNDRICK. 1996. Structure of and relationships within and between the littoral, rock-substrate fish communities off four islands in the Canarian Archipelago. Mar. Biol., 125: 215-231.

FEITOSA, CV, DAS PIMENTA \& ME ARAÚJO. 2002. Ictiofauna recifal dos parrachos de Maracajaú (RN) na área dos flutuantes: inventário e estrutura da comunidade. Arquivo de Ciências do Mar, 35:39-50.
FERREIRA, BP \& M MAIDA. 2006. Monitoramento dos Recifes de Corais do Brasil, situação atual e perspectivas. Ministério do Meio Ambiente (MMA), $120 \mathrm{p}$.

FLOETER, SR, MD BEHRENS, CEL FERREIRA, MJ PADDACK \& $\mathrm{MH}$ HORN. 2005. Geographical gradients of marine herbivorous fishes: patterns and processes. Mar. Biol., 147: 1435-1447.

FLOETER, SR, CEL FERREIRA, A DOMINICI-AROSEMENA \& I ZALMON. 2004. Latitudinal gradients in Atlantic reef fish communities: trophic structure and spatial use patterns. J. Fish Biol., 64: 1680-1699.

FLOETER, SR, BS HALPERN \& CEL FERREIRA. 2006. Effects of fishing and protection on Brazilian reef fishes. Biol. Conserv., 128: 391 - 402 .

FLOETER, SR, RZP GUIMARÃES, LA ROCHA, CEL FERREIRA, CA RANGEL \& JL GASPARINI. 2001. Geographic variation in reef-fish assemblages along the Brazilian coast. Glob. Ecol.Biog., 10: 423-433.

FLOETER, SR \& A SOARES-GOMES. 1999. Biogeographic and species richness patterns of Gastropoda on the southwestern Atlantic. Rev. Bras. Biol., 59: 567-575.

FOSTER, MS. 2001. Rhodoliths: between rocks and soft places. J. Phyc., 37: 659-667.

FRANCINI-FILHO, RB \& RL MOURA. 2008a. Dynamics of fish assemblages on coral reefs subjected to different management regimes in the Abrolhos Bank, eastern Brazil. Aqua. Conser. Mar.Fresh. Ecos., 18: 1166-1179

FRANCINI-FILHO, RB \& RL MOURA. 2008b. Evidence for spillover of reef fishes from a no-take marine reserve: An evaluation using the before-after control-impact (BACl) approach. Fish. Res., 93: 346-356.

FRIEDLANDER AM, EK BROWN, PL JOKIEL, WR SMITH,KS RODGERS. 2003. Effects of habitat, wave exposure, and marine protected area status on coral reef fish assemblages in the Hawaiian archipelago. Coral Reefs, 22:291-305.

FROESE, R \& D PAULY. 2005. eds. Fish Base. World Wide Web electronic publication www.fishbase.org, version (07/2005).

GELL, FR \& CM ROBERTS. 2003. Benefits beyond boundaries: the fishery effects of marine reserves. Tren. Ecol. Evol. 18:448-455.

GHERARDI, DFM. 2004. Community structure and carbonate production of a temperate rhodolith bank from Arvoredo Island, southern Brazil. Braz. J. Ocean., 52(3/4):207-224.

GIBRAN, F. Z. 2007. Activity, habitat use, feeding behavior, and diet of four sympatric species of Serranidae (Actinopterygii: Perciformes) in southeastern Brazil. Neot. Ichth., 5(3): 387-398.

GODOY, E. A. S, GERHARDINGER L. C., DAROS F. \& HOSTIMSILVA, M. 2006. Utilization of Bottom Trawling and Underwater Visual Census Methodologies on the Assessment of the Fish Communities From Arvoredo Biological Marine Reserve, SC, Brazil. J. Coas. Res., Special Issue 39: 1205-1209.

GUIMARÃES, RZP, JL GASPARINI, LA ROCHA, SR FLOETER, CA RANGEL, GWC NUNAN. 2001. Peixes recifais brasileiros: riqueza desconhecida e ameaçada. Ciência Hoje. Vol. 28. no 168, p. 16-23.

HAIR JR, JF, RE ANDERSON, RL TATHAN \& WC BLACK. 1998. Multivariate Data Analysis. Fifth Edition, Prentice Hall. 730p.

HALPERN, BS. 2003. The impact of marine reserves: Do reserves work and does reserve size matter? Ecol. Appl., 13: S117S137 Suppl. S. 
HEITHAUS, MR, A FRID, AJ WIRSING \& B WORM. 2008. Predicting ecological consequences of marine top predator declines. Tren. Ecol. Evol., 23: 202-210.

HOSTIM-SILVA, M, AB ANDRADE, LF MACHADO, LC GERHARDINGER, FA DAROS, JP BARREIROS \& EA GODOY. 2006. Peixes de Costão Rochoso de Santa Catarina: I Arvoredo. Itajaí: Editora UNIVALI, 2005. 135p

HUMANN, P \& N DELOACH. 2003. Reef Fish identification. Florida, Caribbean, Bahamas. Florida, New World Publications. Inc. Florida, USA. 481p.

IBAMA. 2004. Plano de manejo Reserva Biológica Marinha do Arvoredo.

KOETTKER, AG \& AS FREIRE. 2006. The Spatial-Temporal Variation of Decapod Larvae in the Subtropical Waters of the Arvoredo Archipelago, SC, Brazil. Iheringia, 96 (1): 31-39.

LERNER, CB. 1996. Esponjas da Ilha da Galé, Reserva Marinha Biológica da llha do Arvoredo, Santa Catarina, Brasil (Porifera; Desmospongiae). Biociências, 4 (2): 101-129.

LINCOLN SMITH, MP. 1988. Effects of observer swimming speed on sample counts of temperate rocky reef fish assemblages. Mar. Ecol. Prog. Ser., 43: 223-231.

LUBCHENCO, J, SR PALUMBI, SD GAINES \& S ANDELMAN. 2003. Plugging a hole in the ocean: the emerging science of marine reserves. Ecol. Appl., 13 (1): 8-25.

MAGURRAN, AE. 1988. Ecological diversity and its measurement. London, Croon Helm, 179p.

MANLY, BFJ. 1997. Randomization, bootstrap and Monte Carlo methods in biology. Chapman \& Hall, New York. 398p.

MEDEIROS, RP, SC VIZINHO, CX MACEDO \& M POLETTE. 1997. Diagnóstico sócio-econômico e cultural das comunidades pesqueiras artesanais do litoral centro-norte do Estado de Santa Catarina. Notas técnicas da FACIMAR/UNIVALI, 1: 33-42.
MICHELI, F, BS HALPERN, LW BOTSFORD \& RR WARNER. 2004. Trajectories and correlates of community change in notake marine reserves. Ecol. Appl., 14: 1709-1723.

NRC (National Research Council). 2001. Marine protected areas: tools for sustaining ocean ecosystems. Washington, DC, National Academy Press, 272p.

PIELOU, EC. 1974. Population and communication ecology: Principles and Methods. London, Gordon and Breach Science. 424p.

PIMENTA, FM, E MELO, D FRANCO \& PO ZAVIOLOV. 2004. Assessment of Santa Catarina shelf currents through the analysis of indirect measurements. J. Coast. Res., SI 36: 651-655

PIOLA, AR, RP MATANO, ED PALMA, OO MOLLER \& EJD CAMPOS. 2005. The influence of the Plata River discharge on the western South Atlantic shelf. Geophy. Res. Let., 32, L01603.

ROBERTS, CM, JP HAWKINS \& FR GELL. 2005. The role of marine reserves in achieving sustainable fisheries. P. Trans. R. Soc. B, 360: 123-32.

SHANNON, CE \& W WEAVER. 1949. The Mathematical Theory of Communication. University of Illinois Press, Urbana, Illinois. 163p.

SMITH, CL. 2004. National Audubon Society. Field guide to tropical marine fishes of the Caribbean, the Gulf of México, Florida, the Bahamas, and Bermuda. New York, Alfred A. Knopf. 718p.

WIGGERS, F \& AR MAGALHÃES. 2003. Novas ocorrências de moluscos marinhos na Reserva Biológica Marinha do Arvoredo. Biotemas, 16: 81-89.

ZAR, JH. 1984. Biostatiscal Analysis. $2^{\circ}$ edition. New Jersey, Prentice Hall. 718p.

Recebido - 07/05/2009

Aceito: 20/04/2010 